\title{
In Situ Observation of the Effect of Synthesis Conditions on the Growth Rates and Mechanisms of Carbon Nanotubes
}

\author{
Renu Sharma, ${ }^{*}$ Peter Rez ${ }^{* *}$ and M.M.J. Treacy ${ }^{* *}$ \\ ${ }^{*}$ Center for Solid State Science, Arizona State University, Tempe, AZ 85287-1704 \\ Dept. of Physics and Astronomy, Arizona State University, Tempe, AZ 85287
}

Carbon nanotubes have attracted much attention since their discovery because of their remarkable properties [1, 2]. However, the selective synthesis of nanotubes with a desired structure and property is still a challenge. Moreover, for some applications, such as field emitters, they need to be synthesized directly as part of the device or the circuit. In order to determine the best synthesis conditions for a certain type of CNT, it is important to understand the relationship between the synthesis conditions (temperature and pressure) and their structure and properties. Much can be learned by making in situ TEM observations of the nucleation and growth of CNT as they are formed $[3,4]$.

We have observed the CNT nucleation and growth mechanisms using a specially modified environmental transmission electron microscope (ETEM), a Tecnai F20, operated at 200KV and equipped with a Gatan imaging filter (GIF) and annular dark-field detector [5]. $\mathrm{Ni} / \mathrm{SiO}_{2}$ catalyst samples were dry loaded on $\mathrm{Ni}$ grids and heated up to $400-700^{\circ} \mathrm{C}$ in the microscope vacuum $\left(10^{-6}\right.$ Torr) using a furnace heating holder. 10-100 mTorr of acetylene $\left(\mathrm{C}_{2} \mathrm{H}_{2}\right)$ was slowly leaked into the sample region at the reaction temperature, and bright-field images were recorded using a digital video recorder connected to a TV rate camera. The growth rates and growth mechanisms were obtained by studying sequential video frames.

Figures 1a-i show digitized individual frames from an ETEM video sequence recorded at $480^{\circ} \mathrm{C}$ and in 20 mTorr of $\mathrm{C}_{2} \mathrm{H}_{2}$. A number of CNTs were formed during the time lapsed between the introduction of the gas into the specimen area and the time when video recording was started (a few seconds) (Figure 1a). A Ni catalyst particle, marked by the arrow in the first frame (Figure 1a), was observed to move out of the substrate $\left(\mathrm{Ni} / \mathrm{SiO}_{2}\right.$ powder $)$ to form the apex of a hollow tubular structure (Figure 1b). After growing a distance of $\sim 12 \mathrm{~nm}$ in $0.133 \mathrm{sec}$, no further growth occurs in the following $0.1 \mathrm{sec}$ (Figure 1c and Figure 2). Growth then resumed at sharp angles relative to the original growth direction (Figure 1d) and its progression was followed from subsequent time resolved images (Figure 1c-1i). A growth profile was sgenerated (Figure 2) and the incremental change in projected length during each frame $(1 / 30 \mathrm{sec})$ was used to estimate instantaneous growth rates for each frame. The growth rates measured in this manner were broadly distributed, and indicative of discontinuous growth. For example, there is little apparent growth between Figure $1 \mathrm{~b}$ and $1 \mathrm{c}$, and a stagnant period with less than $0.8 \mathrm{~nm}$ of growth in $0.17 \mathrm{~s}$ was observed between Figure 1f-g (see also Figure 2). Instantaneous (projected) growth rates in excess of $100 \mathrm{~nm} / \mathrm{s}$ were observed in numerous frames. At pressures above 20 mTorr of acetylene, CNTs grow in a zigzag manner, bending by $\approx 60^{\circ}$ or $120^{\circ}$, with the apex frequently returning to the substrate to become anchored at both ends. Density functional theory calculations show that such bending could be due to CNT growing on $\mathrm{Ni}\{110\}$ surfaces and will be structurally accommodated by forming pentagon and heptagon defect pairs (Figure 3). SWCNTs were observed to form either at higher temperature $(700 \mathrm{C})$ as reported earlier [3] or at lower temperatures and low pressures $(480 \mathrm{C}$ and below 20 mTorr) (Figure 4). Our data show that the growth rates, duration of growth, and length of the CNTs formed, are controlled by the synthesis conditions. For example, total growth time of individual CNTs after nucleation was observed to be longer at lower gas pressures (15-45 sec compared to 3-5 sec at high gas pressures). At gas pressures above 20 mTorr, nanotubes grew up to $340 \mathrm{~nm}$ in length, in a serpentine manner while straight tubes grew at low pressures ( $\sim 10 \mathrm{mTorr})$ with an average length of $\sim 50 \mathrm{~nm}$. Thus we have been able to obtain a better understanding of the relationship between synthesis conditions and CNT nucleation and growth mechanism that will be discussed in detail. 

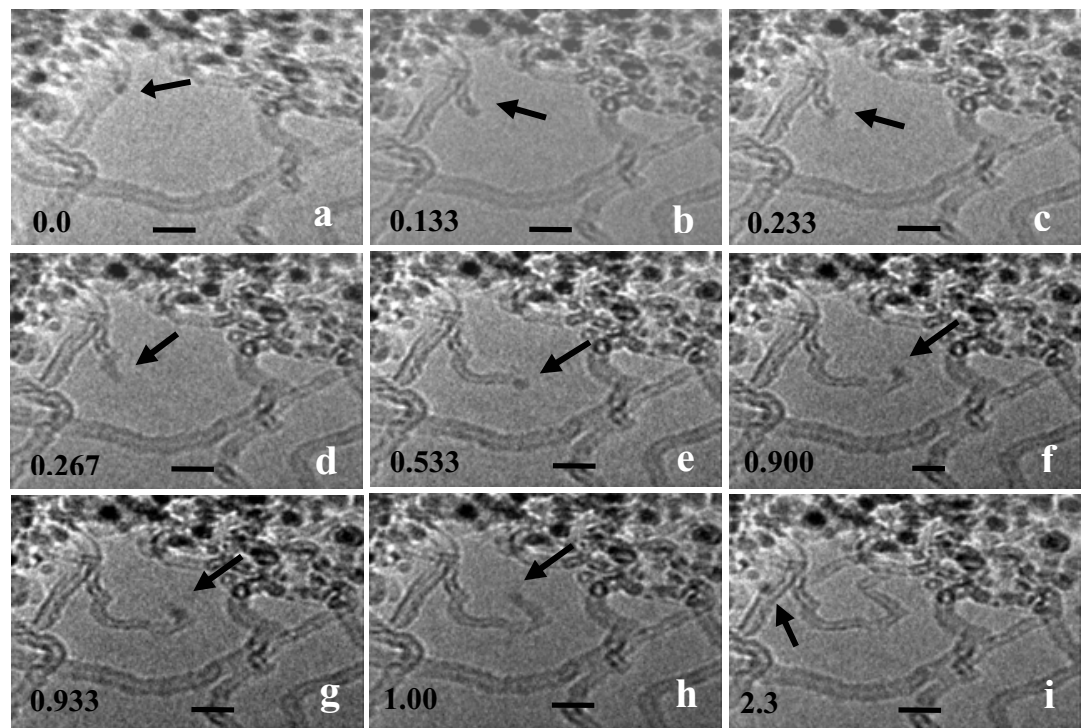

Figure1. Individual frames digitized from a video sequence recorded at $480^{\circ} \mathrm{C}$ in 20 mTorr of $\mathrm{C}_{2} \mathrm{H}_{2}$, showing the nucleation and growth of a carbon nanotube. The apex of the growing CNT is marked by the arrows (a-i). Note the absence of growth between $b$ $c$ and $f-g$. The growth direction changed after each stagnant period and the tube grew in a serpentine, or zig-zag, manner, $(e-h)$ and finally attaching back to the substrate to form a loop (i). The time lapsed from (a) is shown in bottom left corner of each frame, and the bar is $10 \mathrm{~nm}$.

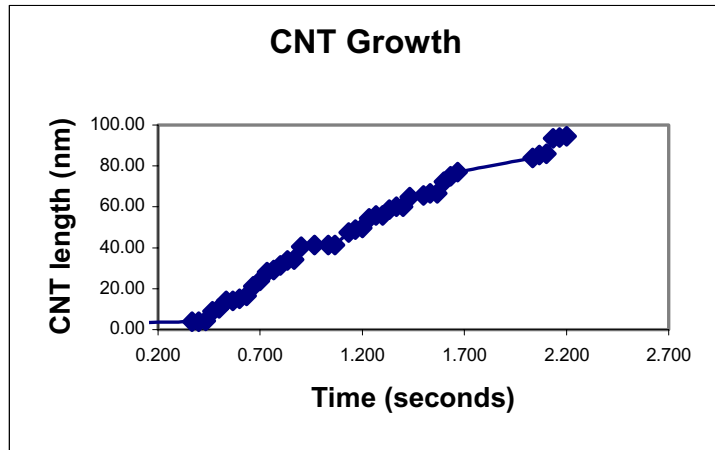

Figure2. Measured length of one CNT is shown as a function of time. The growth rate has discontinuities, ranging from 0 to $100 \mathrm{~nm} / \mathrm{sec}$.

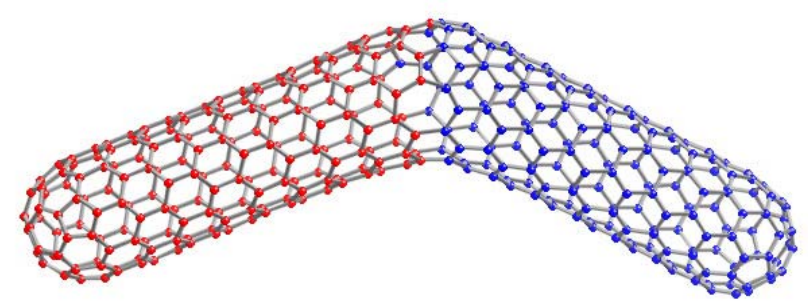

Figure3. A hypothetical bend in a SWCNT induced by three 5ring/7-ring pairs. The tube on the left is a 10,0 nanotube. That on the right is 5,5 nanotube. Despite the bend and the rounded endcaps, the nanotube remains a seamless 3-coordinated structure. The distribution of 5-rings and 7-rings shown here is not unique, many different variants that support such a bend being possible.

\section{References:}

[1] S. Iijima, Nature, 354 (1991) 56.

[2] Dresselhaus,M., Dresselhaus, G., \& Avouris, Ph., Carbon Nanotubes: Synthesis, Structure, Properties and Applications, (2001) Springer-Verlag, Berlin.

[3] Renu Sharma and Zafar Iqbal, Appl Phys. Lett. 84 (2004) 990.

[4] Helveg et al, Nature 427 (2004) 426.

[5] Renu Sharma et al, Microsc. Microanal. (2003) CD314.

[6] Financial support from Arizona State University and use of the John Cowley Center for High Resolution Electron Microscopy is gratefully acknowledged.

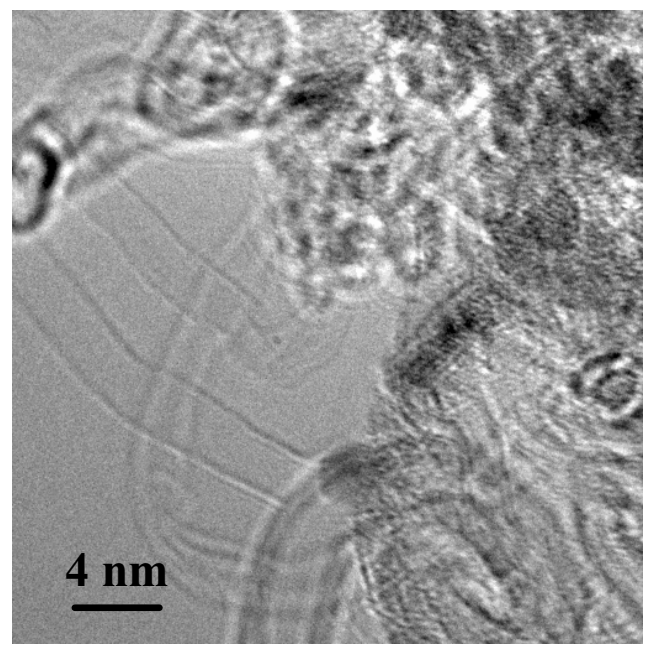

Figure4. High resolution image showing singlewall carbon nanotubes formed at $480^{\circ} \mathrm{C}$ in 20 mTorr during in-situ observations. 